\title{
CALCULATION OF UPSETTING FORCE IN FLASH BUTT WELDING OF CLOSED-SHAPE PRODUCTS
}

\author{
P.N. CHVERTKO, A.V. MOLTASOV and S.M. SAMOTRYASOV \\ E.O. Paton Electric Welding Institute, NASU \\ 11 Bozhenko Str., 03680, Kiev, Ukraine. E-mail: office@paton.kiev.ua
}

\begin{abstract}
In structure elements of flying vehicles the ring-type parts of high-alloy steel and alloys are widely applied. To manufacture such structure elements different methods of fusion welding are applied including flash butt welding (FBW). Technology of FBW of such parts requires high upsetting speeds $\geq 50 \mathrm{~mm} / \mathrm{s}$ with a specific force of not less than 120-200 MPa. In FBW of parts of a closed shape it is important to determine the value of necessary upsetting force. At unlimited or insufficient upsetting force the necessary conditions for joint formation in a solid phase will not be provided, and during overheating the part shortening will be rather large. Both conditions will lead to violation of shape of ring-type parts, in particular, in perimeter. In the process of welding the elastic forces appear in product being welded, hindering the joint formation, which should be determined in the development of technology and equipment for welding. Besides, in welding of ring-type billets of a large section, which are characterized by a significant elasticity, it is necessary to take into account the possibility of a welded butt rupture due to elasticity of billet at insufficient time of upsetting without passing of electric current. Calculation of the above-mentioned forces was carried out according to the theory of thin rods under the conditions, when stress-strain state was considered to be linear. Also the calculation of given forces using the methods of elasticity theory was carried out, and as a result the plain problem of elasticity theory was solved. The comparison of results of calculation of eight rings with different characteristic sizes showed that maximum difference of values of upsetting force, calculated by the theory of thin rods and formulae of plane problem, was about $2 \%$, and the values of forces, rupturing a butt after welding, was less than $1 \%$. Moreover, in decrease of ratio of external radius of the ring to its internal radius the accuracy of calculation according to the theory of thin rods is increased. 7 Ref., 1 Table, 4 Figures.
\end{abstract}

$\boldsymbol{K} \boldsymbol{e} \boldsymbol{y} \boldsymbol{w} \boldsymbol{o} \boldsymbol{d} \boldsymbol{s}:$ flash butt welding, rings, high-alloyed steels, stress-strain state, upsetting force, theory of thin rods, plain problem

In industry, to manufacture the parts and structures of a closed shape of different purpose (elements of engines, different bandages, turntables, bearings, flanges, etc.), different methods of welding are applied (arc, electron beam, flash

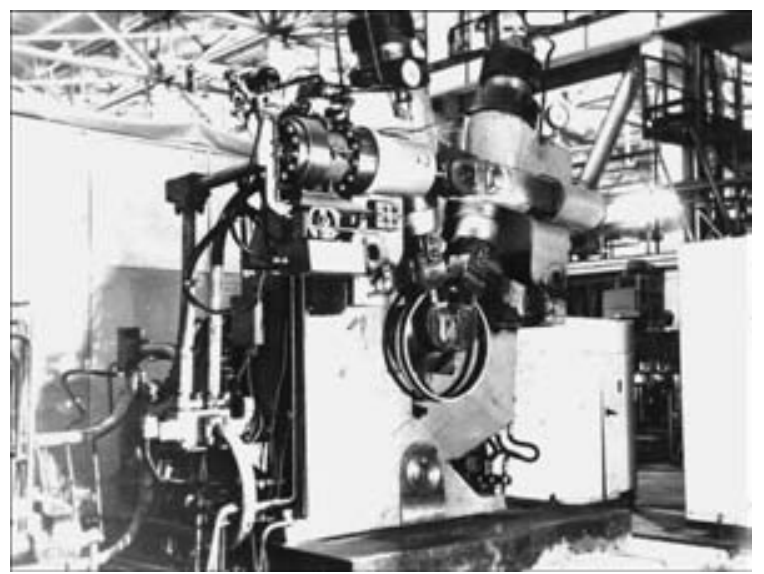

Figure 1. Ring-type billet in the clamping devices of butt welding machine K607

(C) P.N. CHVERTKO, A.V. MOLTASOV and S.M. SAMOTRYASOV, 2014 butt, etc.) on which the service characteristics of welded joint greatly depend.

One of the most saving and technically simple processes of manufacture of ring-type billets is FBW [1]. This method provides high stable quality of welded joints, unites welding and assembly operations in one cycle and does not require application of auxiliary consumables (electrodes, fluxes, welding wires, shielding gases, etc.).

At the E.O. Paton Electric Welding Institute the technology and equipment [2] for FBW of ring-type parts under the industrial conditions were developed (Figure 1). The technology was based on the method of FBW with pulsating flashing [3], which provides the highly-concentrated heating, due to which the HAZ is decreased.

The calculation diagram of loading the ring, on which the upsetting force $P$ and reactive moment $M$ from the movable clamping device of the machine, corresponding to Figure 1, are affected, is given in Figure 2.

It can be assumed as the first approximation that the sizes of the cross section of the ring are 
small as compared to its average radius, then the problem can be solved using the thin rods theory.

To determine forces $P$ it is rationally to relate it with the value of elastic movement $\delta_{\mathrm{el}}$ from the initial gap up to the final position considering the shortening of part due to flashing and upsetting. The value of elastic movement in a curvilinear contour should be determined using More integral:

$$
\delta_{\mathrm{el}}=\int_{S} \frac{\bar{M} M_{p} d s}{E I_{z}},
$$

where $\bar{M}$ is the expression for moment from single force applied in the direction of force $P ; M_{p}$ is the expression for the moment from active and reactive forces; $I_{z}$ is the moment of section inertia; $E$ is the elasticity modulus of the first kind.

Using canonic equations of method of forces [4], the value of reactive moment $M=P(a+$ $+b) / 2$ was found. Considering that the expression for bending moment in ring sections has a form

$$
M_{p}=\frac{1}{2} P(a+b) \cos \theta .
$$

Expression for determination of moment from action of single force $\bar{P}=1$ will be written in a form

$$
\bar{M}=\frac{1}{2}(a+b)(1-\cos \theta) .
$$

Substituting expressions (2) and (3) into (1), the value of elastic movement was determined:

$$
\delta_{\mathrm{el}}=\frac{1}{E I_{z}} \times
$$

$\times\left[2 \int_{0}^{\pi} \frac{1}{2} P(a+b) \cos \theta \frac{1}{2}(a+b)(1-\cos \theta) \frac{1}{2}(a+b) d \theta\right]=$

$$
=\frac{\pi P(a+b)^{3}}{8 E I_{z}},
$$

from which it is possible to obtain the upsetting force

$$
P=\frac{8 E I_{z}}{\pi(a+b)^{3}} \delta_{\mathrm{el}} .
$$

We obtain the more precise solution of this problem by solution of plane problem of theory of elasticity. This solution belongs to Kh.S. Golovin and is described in work [5]. Therefore, we give only expressions for stresses:

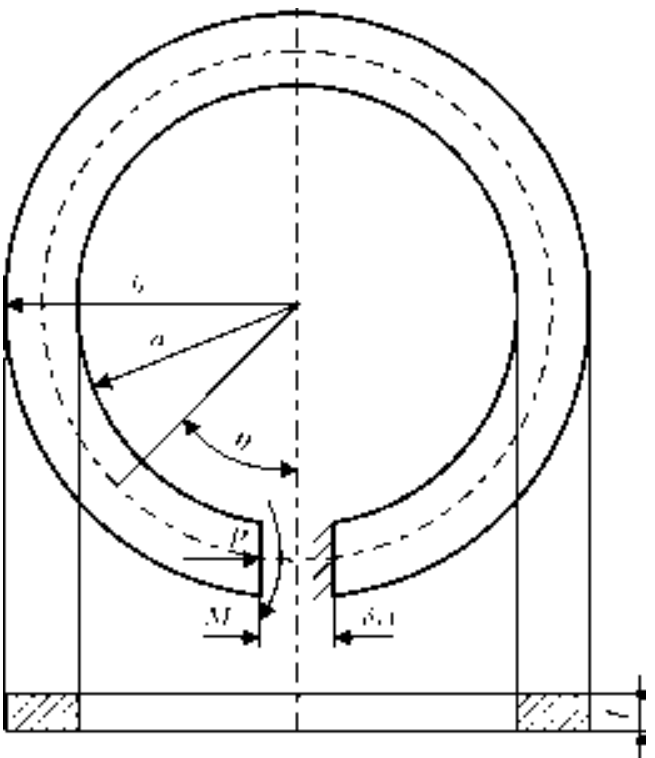

Figure 2. Schematic of ring loading in FBW

$$
\left\{\begin{array}{l}
\sigma_{r}=\frac{\partial \varphi}{r \delta r}+\frac{\delta^{2} \varphi}{r^{2} \partial \theta^{2}}=\left(2 A r-\frac{2 B}{r^{3}}+\frac{D}{r}\right) \cos \theta, \\
\sigma_{\theta}+\frac{\partial^{2} \varphi}{\delta r^{2}}=\left(6 A r+\frac{2 B}{r^{3}}+\frac{D}{r}\right) \cos \theta, \\
\tau_{r \theta}=-\frac{\partial}{\partial r}\left(\frac{\partial \varphi}{r \partial \theta}=\left(2 A r-\frac{2 B}{r^{3}}+\frac{D}{r}\right) \sin \theta,\right.
\end{array}\right.
$$

where $A, B$ and $D$ are the constants determined by the boundary conditions. In our case they are equal:

$$
A=\frac{-P}{2 R} ; \quad B=\frac{P a^{2} b^{2}}{2 R} ; \quad D=\frac{P}{R}\left(a^{2}+b^{2}\right) .
$$

Moreover

$$
R=a^{2}-b^{2}+\left(a^{2}+b^{2}\right) \ln \frac{b}{a} .
$$

Unlike work [5] where during study of movements the functions of complex variable were used, we used the method, which was applied in [6] for solution of problem about the action of tangent force applied at the end of a curvilinear rod.

The components of deformation in the polar coordinates has the following form:

$$
\varepsilon_{r}=\frac{\partial u}{\partial r} ; \quad \varepsilon_{\theta}=\frac{u}{r}+\frac{\partial v}{r \partial \theta} ; \quad \gamma_{r \theta}=\frac{\partial u}{r \partial \theta}+\frac{\partial v}{\partial r}-\frac{v}{r} .
$$

Through the components of stress (5) the components of deformation in plain-stressed state are expressed in the following way:

$$
\varepsilon_{r}=\frac{1}{E}\left(\sigma_{r}-\mu \sigma_{\theta}\right) ; \varepsilon_{\theta}=\frac{1}{E}\left(\sigma_{\theta}-\mu \sigma_{r}\right) ; \gamma_{r \theta}=\frac{\tau_{r \theta}}{G},
$$

where 


$$
G=\frac{E}{2(1+\mu)} .
$$

Having compared the expressions for $\varepsilon_{r}$ from (7) and (8) considering (5), we obtained after integration

$$
\begin{gathered}
u=\frac{\cos \theta}{E} \times \\
\times\left[A r^{2}(1-3 \mu)+\frac{B}{r^{2}}(1+\mu)+D(1-\mu) \ln r\right]+f(\theta),
\end{gathered}
$$

where $f(\theta)$ is the function depending only on the argument $\theta$, which will be determined below. By substitution of obtained expression (9) into expression for $\varepsilon_{\theta}$ from (7) considering (8) we shall obtain after integration

$$
\begin{gathered}
v=\frac{\sin \theta}{E} \times \\
\times\left[A r^{2}(5+\mu)+\frac{B}{r^{2}}(1+\mu)+D(1-\mu)(1-\ln r)\right]- \\
-\int f(\theta) d \theta+F(r),
\end{gathered}
$$

where $F(r)$ is the function depending only on the argument $r$, which also will be determined below. Substituting obtained expression (10) together with expression (9) into expression for $\gamma_{r \theta}$ from (7) considering (8) we shall obtain

$$
\frac{4 D \sin \theta}{E}=r \frac{d F}{d r}+\int f(\theta) d \theta-F(r)+\frac{d f(\theta)}{d \theta},
$$

which should comply with functions $f(\theta)$ and $F(r)$. These functions were determined by the authors in the following form:

$$
f(\theta)=\frac{2 D}{E} \theta \sin \theta+K \sin \theta+L \cos \theta ; F(r)=H r
$$

where $K, L, H$ are the arbitrary constants determined from the conditions of clamping.

Considering (11) the expressions for components of movement have a form

$$
\left\{\begin{array}{l}
u=\frac{2 D}{E} \theta \sin \theta+\frac{\cos \theta}{E} \times \\
\times\left[A r^{2}(1-3 \mu)+\frac{B}{r^{2}}(1+\mu)+\right. \\
+D(1-\mu) \ln r]+K \sin \theta+L \cos \theta \\
v=\frac{2 D}{E} \theta \cos \theta+\frac{\sin \theta}{E} \times \\
\times\left[A r^{2}(5+\mu)+\frac{B}{r^{2}}(1+\mu)+\right. \\
+D[(1-\mu)(1-\ln r)-2]]+K \cos \theta-L \sin \theta+H r .
\end{array}\right.
$$

Tangential movement of a free end can be obtained by substituting value $\theta=0$ into expression for $v$, then

$$
v(\theta=0)=K+H r .
$$

Constants $K, H$ are determined from the conditions on stationary fixed end $\theta=2 \pi$, where $v=$ $=0, \partial v / \partial r=0$. Substituting that into expression for $v$ from (12) the unknown constants were determined:

$$
K=-\frac{4 \pi D}{E} ; \quad H=0 .
$$

Thus, using formulae (6) we will find at $\theta=0$

$$
v=\delta_{\mathrm{el}}=-\frac{4 \pi P\left(a^{2}+b^{2}\right)}{E\left[a^{2}-b^{2}+\left(a^{2}+b^{2}\right) \ln \frac{b}{a}\right]} .
$$

From formula (15) we shall determine the upsetting force:

$$
P=\frac{E \delta_{\mathrm{el}} t}{4 \pi\left(a^{2}+b^{2}\right)}\left[a^{2}-b^{2}+\left(a^{2}+b^{2}\right) \ln \frac{b}{a}\right] .
$$

The character «-» evidences that direction of movement $v$ does not coincide with the direction of growth $\theta$. It should be noted that thickness $t$ was introduced to formula (16) as for the solution of plane problem we would obtain the force per a unit of thickness. Rejects and defects in welding are mainly detected after operation of calibration or in the process of subsequent mechanical treatment. Those rings can be considered defective, in which during removal of upsetting force the cracks are formed in a butt or fracture along the welded butt is occurred.

During determination of force tending to rupture the butt joint after welding, it is necessary to consider that during removal of the upsetting force the reactive moment stops acting as far as clamping device creating this reaction is open. Thus, when a weldement is trying to obtain the previous geometric shape, the stressed state is predetermined only by the effect of the force $P$, directed to the opposite direction to the upsetting force (Figure 3 ).

To solve this problem by the theory of thin rods it is sufficient to substitute into expression (1) instead of (2)

$$
M_{p}+\frac{1}{2} P(a+b)(1-\cos \theta) .
$$

Then we shall obtain 


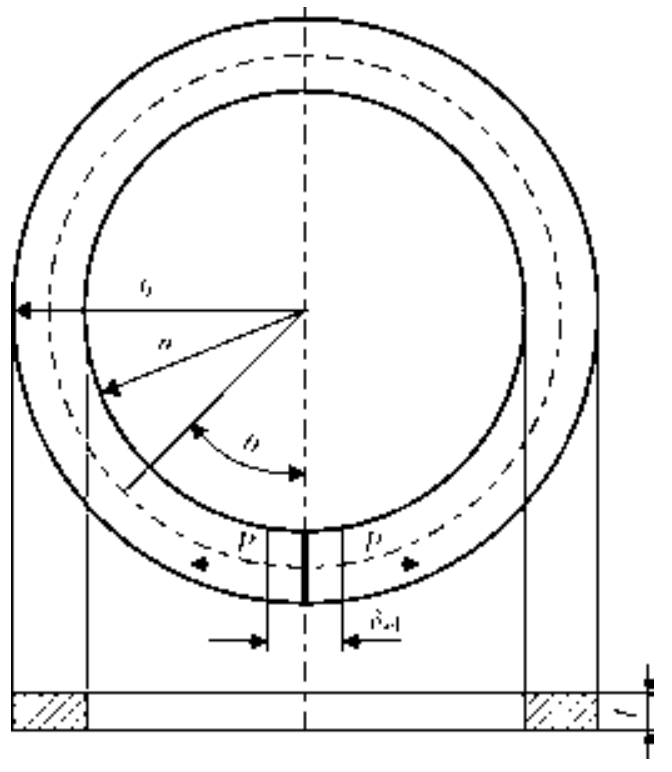

Figure 3. Schematic of ring loading after welding

$$
\begin{gathered}
\delta_{\mathrm{el}}=\frac{1}{E I_{z}} \times \\
\times\left[1 \int_{0}^{\pi} \frac{1}{2} P(a+b)(1-\cos \theta) \frac{1}{2}(a+b)(1-\cos \theta) \frac{1}{2}(a+b) d \theta\right]= \\
=\frac{3 \pi P(a+b)^{3}}{8 E I_{z}},
\end{gathered}
$$

from where it is possible to obtain the force, tending to rupture the butt joint after welding:

$$
P=\frac{8 E I_{z}}{3 \pi(a+b)^{3}} \delta_{\mathrm{el}} .
$$

As is seen from expression (18), in the frames of theory of thin rods the force $P$, tending to rupture the butt joint after welding, is always 3 times less than the upsetting force determined by formula (4).

It should be noted that formula (18) coincides with formula, obtained in work [7], in another way. To solve this problem using methods of theory of elasticity it is necessary to subtract stresses,

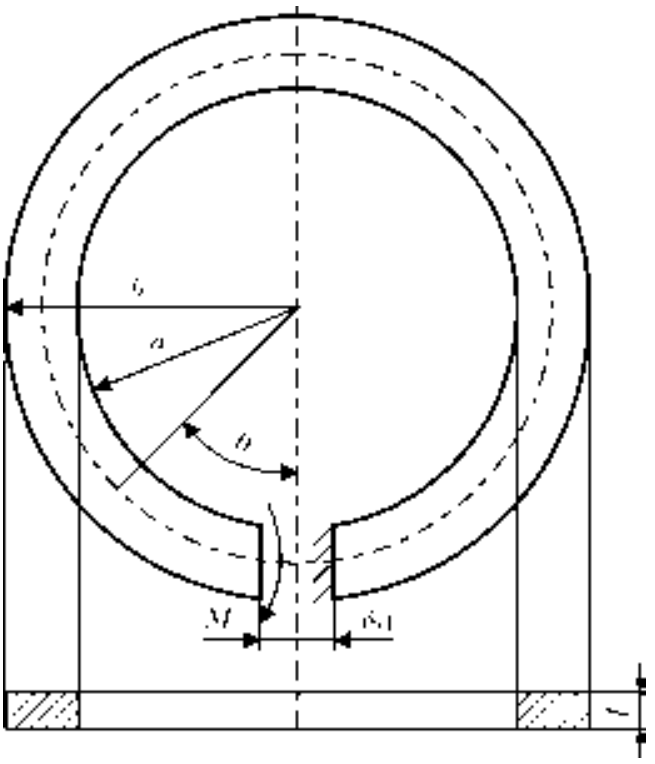

Figure 4. Calculation scheme of ring under bending by moment in curvature plane

arising under the action of moment, from expressions (5) (Figure 4):

$$
v=\frac{8 \pi M(a+b)\left(b^{2}-a^{2}\right)}{E\left[\left(b^{2}-a^{2}\right)^{2}-4 a^{2} b^{2}\left(\ln \frac{b}{a}\right)^{2}\right]} \text { at } \theta=2 \pi .
$$

The solution of this problem also belongs to Kh.S. Golovin and is available in literature [5, 6].

The value of elastic deformation $\delta_{\mathrm{el}}$ consists of movement, given by formula (15), taken with an opposite sign, and movements given by formula (19). Taking into account that $M=P(a+$ $+b) / 2$ and multiplying by thickness $t$, we shall obtain expression for the force acting on the butt joint after welding

$$
P=\frac{E \delta_{\mathrm{el}} t}{4 \pi N}
$$

where

Results of calculation of upsetting forces and forces rupturing butt joint after welding

\begin{tabular}{|c|c|c|c|c|c|c|c|c|c||}
\hline \hline$a, \mathrm{~mm}$ & $b, \mathrm{~mm}$ & $t, \mathrm{~mm}$ & $\delta_{\mathrm{el}}, \mathrm{mm}$ & $P_{\mathrm{w}}, \mathrm{kN}(4)$ & $P_{\mathrm{w}}, \mathrm{kN}(16)$ & $\Delta P_{\mathrm{w}}, \%$ & $P_{\mathrm{r}}, \mathrm{kN}(18)$ & $P_{\mathrm{r}}, \mathrm{kN}(20)$ & $\Delta P_{\mathrm{r}}, \%$ \\
\hline 160 & 210 & 10 & 13 & 13.62 & 13.48 & 1.08 & 4.54 & 4.52 & 0.52 \\
\hline 180 & 230 & 12 & 14 & 12.94 & 12.83 & 0.88 & 4.31 & 4.29 & 0.43 \\
\hline 255 & 347 & 14 & 20 & 42.44 & 41.86 & 1.37 & 14.15 & 14.05 & 0.67 \\
\hline 243 & 323 & 16 & 19 & 36.45 & 36.03 & 1.18 & 12.15 & 12.08 & 0.57 \\
\hline 241 & 351 & 16 & 22 & 95.89 & 94 & 2.01 & 31.96 & 31.65 & 0.98 \\
\hline 175 & 215 & 22 & 16 & 16.13 & 16.03 & 0.63 & 5.38 & 5.36 & 0.31 \\
\hline 181 & 216 & 25 & 16 & 11.64 & 11.58 & 0.46 & 3.88 & 3.87 & 0.26 \\
\hline 356 & 421 & 25 & 21 & 13.05 & 13 & 0.42 & 4.35 & 4.34 & 0.23 \\
\hline \hline
\end{tabular}




$$
\begin{gathered}
N=\frac{\left(a^{2}+b^{2}\right)}{a^{2}-b^{2}+\left(a^{2}+b^{2}\right) \ln \frac{b}{a}}+ \\
+\frac{\left(a^{2}+b^{2}\right)\left(b^{2}-a^{2}\right)}{\left(b^{2}-a^{2}\right)^{2}-4 a^{2} b^{2}\left(\ln \frac{b}{a}\right)^{2}} .
\end{gathered}
$$

Results of calculations for eight typical rings of steel 12Kh18N10T $(E=200,000 \mathrm{MPa})$, which are used in the structure elements of flying vehicles manufactured at the SE «A.M. Makarov Yuzhny Machine Building Plant», are given in the Table.

In the Table according to results of calculation of upsetting force, made using the theory of thin rods (4) and the method of theory of elasticity (16), maximum deviation $\Delta P_{\mathrm{w}}$ is about $2 \%$. Similarly using formulae (18) and (20) the forces, tending to rupture the butt joint after welding, were calculated, maximum deviation $\Delta P_{\mathrm{r}}$ here was less than $1 \%$. It evidences that in this case the theory of thin rods gives very accurate results. Besides, it can be noted that the error is decreased at decrease of $b / a$ ratio (in fact, if $b$ is tending to $a$, we shall obtain a thin rod and the error will tend to zero).

\section{Conclusions}

1. Using two analytical methods of calculation the expressions for determination of upsetting force necessary in FBW of ring-type billets and the force tending to rupture the butt joint after welding were obtained.

2 . The results of calculations by formulae are given, obtained on the basis of different calculation methods, for eight typical rings. The maximum deviation of value of upsetting force was about $2 \%$ and that for rupture was less than $1 \%$.

3 . The technology and equipment were developed for industrial manufacture of rings of heatresistant alloys and steels of types $12 \mathrm{Kh} 18 \mathrm{~N} 10 \mathrm{~T}$, EI711 and EI712 with the diameter of middle surface from 370 to $777 \mathrm{~mm}$ and $500-1760 \mathrm{~mm}^{2}$ cross section area.

1. Kuchuk-Yatsenko, S.I. (1992) $F B W$. Kiev: Naukova Dumka.

2. Kuchuk-Yatsenko, S.I., Chvertko, P.N., Semyonov, L.A. et al. (2013) FBW of products of high-strength alloys based on aluminium. The Paton Welding J., 7, $2-6$.

3. Kuchuk-Yatsenko, S.I., Didkovsky, V.A., Bogorsky, M.V. et al. Method of resistance butt welding. Pat. 46820 Ukraine. Publ. 17.06.2002.

4. Pisarenko, G.S., Yakovlev, A.P., Matveev, V.V. (1975) Handbook on strength of materials. Kiev: Naukova Dumka.

5. Lurie, A.I. (1970) Theory of elasticity. Moscow: Nauka.

6. Timoshenko, S.P., Goodier, J.N. (1975) Theory of elasticity. Moscow: Nauka.

7. Kochergin, K.A. (1952) Selection of technology of resistance welding. Leningrad: Sudpromgiz.

Received 25.08.2013 